\title{
Channel Correlation-based approach for feedback overhead reduction in massive MIMO
}

\author{
Frédéric Challita ${ }^{1}$, Pierre Laly ${ }^{1}$, Marwan Yusuf ${ }^{2}$, Emmeric Tanghe $^{2}$, Wout Joseph ${ }^{2}$, Pierre Degauque ${ }^{1}$, Martine \\ Liénard $^{1}$, Davy P. Gaillot ${ }^{1}$
}

\begin{abstract}
For frequency-division duplex (FDD) Multiple-Input Multiple-Output (MIMO) systems, the channel state information at the transmitter is usually obtained by sending pilots or reference signals from all elements of the antenna array. The channel is then estimated by the receiver and communicated back to the transmitter. However, for massive MIMO, this periodical estimation of the full transfer matrix can lead to prohibitive overhead. To reduce the amount of data, we propose to estimate the updated channel matrix from the knowledge of the full correlation matrix at the transmitter made during some initialization time and the instantaneous measured channel matrix of smaller size, characterizing the link between the user and a limited number of reference array elements. The proposed algorithm is validated with measured massive MIMO channel transfer functions at $3.5 \mathbf{G H z}$ between a $9 \times 9$ uniform rectangular array and different user positions. Since measurements were made in static conditions, the criteria chosen for evaluating the performance of the algorithm is based on a comparison of the predicted channel capacity calculated from either the measured or estimated channel matrix.
\end{abstract}

Index Terms-Index Terms-Massive MIMO, Industry 4.0, Tx Correlation, Channel Estimation.

\section{INTRODUCTION}

$\mathbf{M}$ ASSIVE MIMO is expected to be a cornerstone for the upcoming fifth generation (5G) with multiple use-cases [1] to address new requirements [2] and tackle challenges such as spectral efficiency. Increasing the number of radiating elements at the fixed transmitting side (Tx) in massive MIMO helps alleviate small scale fading effects and decrease interuser correlation [3], [4]. The advantages brought by massive MIMO can benefit industrial automation and machine-tomachine (M2M) communication [5], [6], in the frame of the 4th industrial revolution or Industry 4.0 [7]. For timedivision duplex (TDD) based systems, channel reciprocity is used to estimate the instantaneous channel from uplink (UL) pilots. For frequency-division duplex (FDD), since UL and downlink (DL) use different frequency bands, channel state information at the transmitter (CSIT) is usually obtained by sending orthogonal pilots from all Tx elements and requesting channel feedback from user equipment (UEs). However, for massive systems using a very large number of transmitting

This work was funded through the OS4 SMARTIES research program by the ELSAT2020 project co-financed by the European Union with the European Regional Development Fund, the French state and Hauts de France Region.

F. Challita, D. P. Gaillot, M. Liénard and Pierre Degauque are with the University of Lille, IEMN, Bâtiment P3,Villeneuve d'Ascq, 59655 FR e-mail: frederic.challita@univ-lille.fr.

Emmeric Tanghe ${ }^{2}$, Marwan Yusuf and Wout Joseph ${ }^{2}$ are with Ghent University/IMEC INTEC-WAVES 9052 Gent e-mail: wout.joseph@UGent.be antennas, CSIT may generate high overhead which fundamentally limits the number of potential simultaneous data streams. Various solutions have already been proposed to cope with this problem.

A simplified DL scheduling based on joint spatial division and multiplexing was studied in [8], [9], [10], the UEs being clustered into groups having similar channel covariances. If the distribution of the UEs is such that the channel matrix has a low rank, [11] proposed to use this property to make a joint recovery of CSIT for all scheduled UEs, each of them directly feeding back the pilots observation to the base station (BS) without performing channel estimation. Compressivesensing-based approaches that exploit sparsity properties of the channel are proposed in [12], [13] while temporal-correlation approaches using trellis-code quantization codebooks and memory channel sequences to decrease CSIT estimation are described in [14]. In [15], the basic idea is to exploit the structure of the spatial channel correlation matrix (CCM) with reduced CSIT and to use the dominant eigenspace and Karhunen-Loeve representations. These models are mostly applied to analytical channels (Rayleigh channel) assuming non line-of-sight (NLOS) propagation and mutually independent user channels with rich local scattering. It must be emphasized that results based on measured channels are quite scarce.

In this paper, an easy to implement simple approach to decrease the overhead related to the determination of CSIT is proposed and tested using measured channels in an industrial hall in the context of Industry 4.0. It is based on three successive steps: (i) during the initialization process at time $t$, the full CSIT is measured with the classical approach using feedback of the UEs, allowing the determination of the CCM matrix at the transmitter $\boldsymbol{\rho}_{\mathrm{Tx}}(t)$. (ii) To get an updated estimate of CSIT at time $t+\Delta t$, the channel transfer matrix $\mathbf{H}_{\text {ref }}(t+\Delta t)$ between the UEs and only a small number of array elements, called reference antennas ( ref) in the following, is measured, in order to strongly decrease the overhead time. (iii) An updated estimation $\hat{\mathbf{H}}(t+\Delta t)$ of CSIT is obtained at the BS from the knowledge of $\boldsymbol{\rho}_{\mathrm{Tx}}(t)$ and $\mathbf{H}_{\text {ref }}(t+\Delta t)$. In order to choose the optimum values of the different parameters and to test their performance, massive MIMO radio channels at $3.5 \mathrm{GHz}$ in indoor scenarios were measured rather than simulated propagation channels. Various LOS and NLOS configurations have been considered, the fixed Tx array being a $9 \times 9$ uniform rectangular array (URA).

This letter is organized as follows. In Section II, the configuration of the industrial environment is first described, including the characteristics of the Tx and receiving antenna 
(Rx) and the measurement set-up. Section III presents results on channel correlation at Tx, for all scenarios. The principle of the CSIT estimation algorithm is detailed in Section IV, while the approach to optimally select the reference antennas is described in Section V. Finally, the performance and validity of the algorithm are discussed.

\section{DESCRIPTION OF THE MEASUREMENT CONFIGURATION}

\section{A. Geometrical configuration}

The propagation environment is a large industrial hall located in Technologiepark-Zwijnaarde, Belgium [16]. The $21 \times 77 \times 12 \mathrm{~m}^{3}$ hall is dedicated for testing the robustness of concrete structures. The dominant building material is concrete. The windows are located near the ceiling and a large metallic industrial door closed during measurements, is located at the end of the hall. Metallic machines and measurement tools can be found in the environment, typical for automation cells in Industry 4.0. A panoramic view is given in Fig. 1 (a). The scenario is a multi-user massive MISO (MU-MISO) setup with $K=15$ distributed single-antenna users and whose positions are shown in Fig. 1 (b) by the numbered circles. The machines distributed in the hall can either act as reflectors or block the direct path between Tx and Rx, but are not indicated.

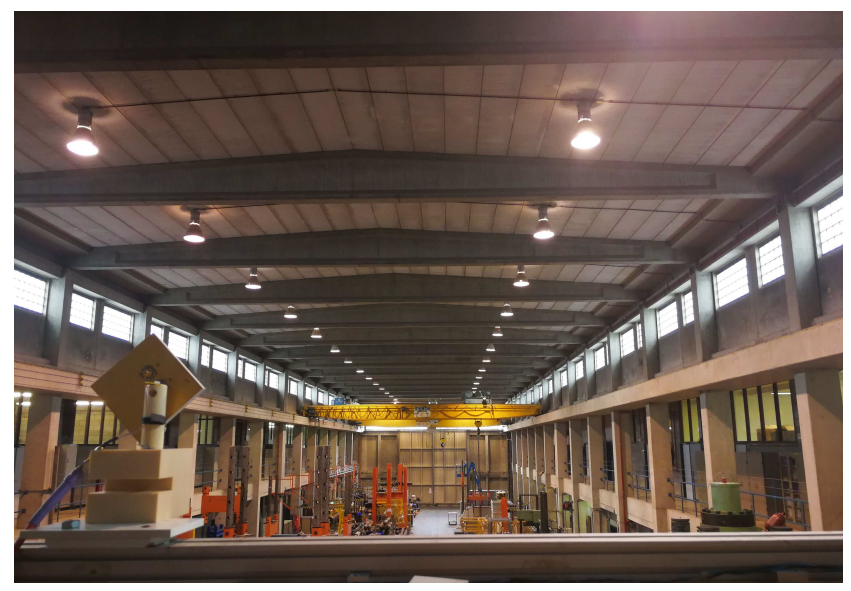

(a)

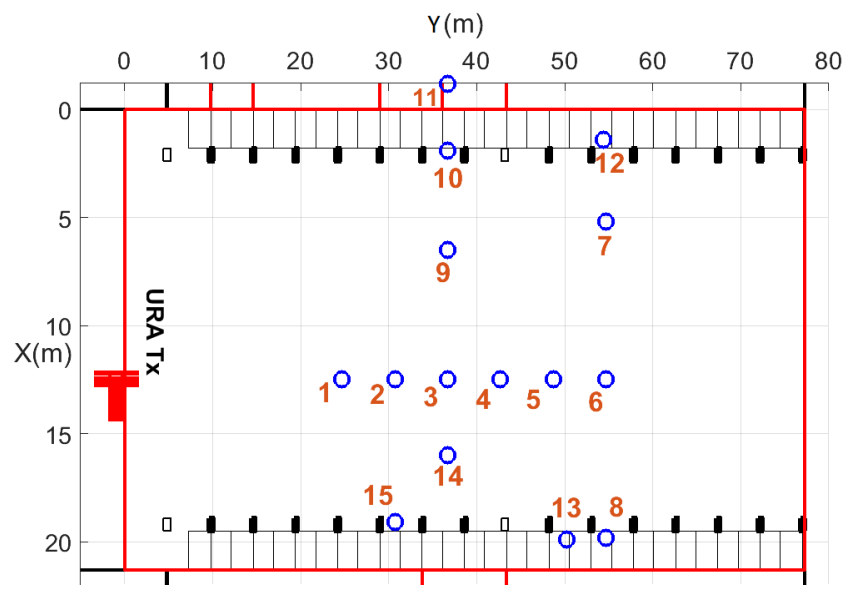

(b)

Fig. 1. a) Panoramic view of the industrial hall from the Tx and $b$ ) Top view showing the positions of the URA and the various Rx positions
The Rx positions have been chosen to cover most of the operational configurations, i.e. LOS (positions 1-2 and 4-7), NLOS (positions 8-11, 14-15) and obstructed LOS (OLOS - positions 3, 12-13). For the last case, the direct path is only masked by a small metallic (or concrete) structure. The horizontal distance between $\mathrm{Tx}$ and the farthest $\mathrm{Rx}$ is 56 $\mathrm{m}$. The transfer matrices were measured by moving the $\mathrm{Rx}$ antenna to each selected position at constant height equal to $1.6 \mathrm{~m}$. The Tx array is a virtual vertical URA of size $M_{\mathrm{x}}=9$ and $M_{\mathrm{z}}=9, M_{\mathrm{x}}$ and $M_{\mathrm{z}}$ being the number of antennas along $\mathrm{x}$ and $\mathrm{z}$ axis. $M_{t}=M_{\mathrm{x}} M_{\mathrm{z}}$ is the total number of antennas. The positioning system of the moving Tx antenna is controlled through a fiber optics link with a dedicated LabView program installed on a Windows PC. The inter-element spacing of the virtual array is $\lambda / 2$ at the center frequency of $3.5 \mathrm{GHz}$ leading to a $38 \mathrm{~cm} \times 38 \mathrm{~cm}$ URA. The mean height above ground being $6.5 \mathrm{~m}$, Tx overlooks the hall as seen in Fig. 11.a).

\section{B. Measurement set-up}

Tx and Rx are vertically polarized patch antennas with an $80 \mathrm{MHz}$ bandwidth $\left(S_{11}<-10 \mathrm{~dB}\right)$. They have an $80^{\circ}$ half power beamwidth, both in azimuth and elevation and a $7 \mathrm{dBi}$ gain. The main beam of $\mathrm{Rx}$ is always oriented towards the Tx array for all studied LOS and NLOS scenarios. Channel measurements were performed in the frequency domain using a vector network analyzer (VNA - Agilent E5071C). The VNA is in the vicinity of Tx and optical fiber optics were deployed for connecting Rx through optical/radio frequency interfaces. Results presented in this letter consider a frequency band of $20 \mathrm{MHz}$, which is the maximum available DL bandwidth for Long Term Evolution (LTE) systems. Within this band, the channel transfer function is measured on $M_{f}=205$ frequency points. Nevertheless, to increase the number of realizations, measurements have been successively made on 4 adjacent 20 $\mathrm{MHz}$ bands allowing us to study the average performance of the proposed CSIT recovery. For each position $k$ of $\mathrm{Rx}$, the wideband complex channel transfer function $\mathbf{h}_{k, i j}(f)$ between $\mathrm{Rx}$ and any element $(i, j)$ of the Tx array is given by the $S_{21}$ scattering parameter measured by the VNA. Considering all $M_{f}$ frequency points, the transfer function becomes a vector $\mathbf{h}_{k, i j} \in \mathbb{C}^{1 \times M_{f}}$.

\section{CORRELATION BETWEEN ARRAY ANTENNAS}

The influence of the antenna element separation on the correlation coefficient $\rho$ has been analyzed for spacing values varying from $d$ to $8 d$. Due to the hall configuration and the relative positions of the Tx and Rx antennas (Fig. 1), $\rho$ varies differently across elements aligned along a vertical line ( $\mathrm{z}$ axis) or a horizontal line ( $x$ axis). CDF (cumulative distribution function) curves corresponding to these 2 cases are shown in Fig. 2(a) and (b), respectively, and are deduced from all $\rho$ values calculated for the $15 \mathrm{Rx}$ positions. 


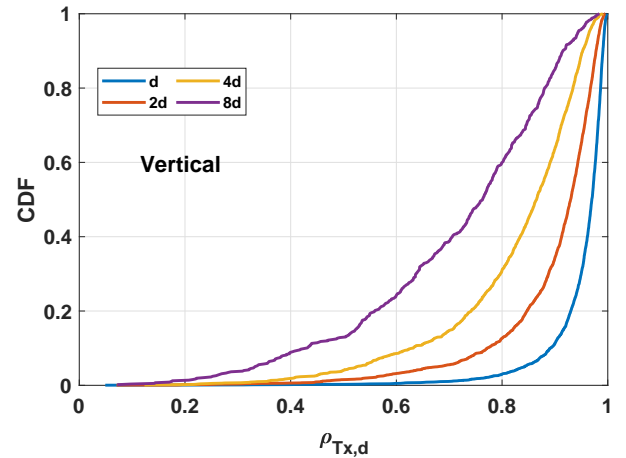

(a)

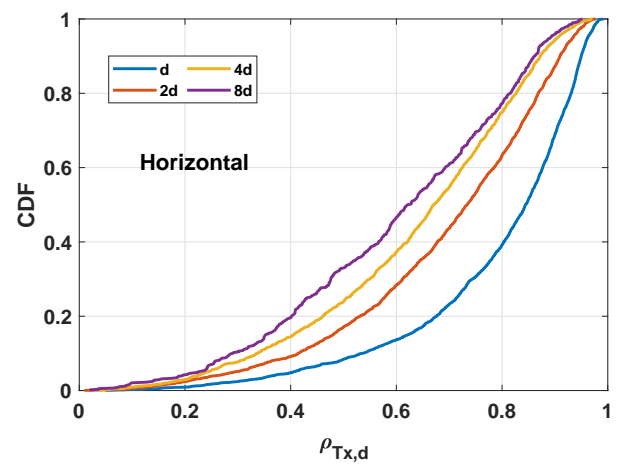

(b)

Fig. 2. CDF of the correlation coefficient for antenna element separation varying from $d$ to $8 d$. Correlation between (a) vertical elements; (b) horizontal elements.

Figs. 2(a) and (b) indicate that $\rho$ is much higher in the vertical plane than in the horizontal plane. Indeed, in this vertical plane, all possible rays have a large elevation angle with small angular spread minimizing the phase shift between antennas. For the horizontal correlation, the median value of $\rho$ decreases as a function of separation, from 0.84 for $d$, to 0.73 for $2 d$ and 0.61 for $8 d$. These results will be used to optimize the parameters of the proposed approach minimizing CSIT overhead.

\section{PRINCIPLE OF THE CSIT ESTIMATION PROCEDURE}

To reduce the amount of notation and without loss of generality, only one $\mathrm{Rx}$ is considered here. Hence, the subscript $k$ identifying the user is omitted. It is assumed that during an initialization process at time $t$, the DL channel transfer vector $\mathbf{h}_{i j}$ between the user and any antenna $(i, j)$ of the array is estimated by the UE (Rx) and sent back to the BS (Tx). The question which arises is to estimate this transfer vector at time $t+\Delta t$, while minimizing the complexity of the procedure and the number of symbols required for this updated estimation. The fundamental idea is to measure the channel between the user and a reduced number of antennas $M_{\text {ref }}$ called reference antennas, belonging to a subset of the massive array, and called reference antennas. The full transfer matrix will be estimated owing to the knowledge of a reduced correlation vector, as detailed in the next paragraph. In order to simplify the notation, the different channel matrices are vectorized using the vec operator.

\section{A. Reduced correlation vector and reduced channel transfer} vector

Let $(i, j)$ be the indices of any Tx antenna, and $\left(i^{\prime}, j^{\prime}\right)$, noted ref those of the reference antenna. The channel vectors between UE and $(i, j)$ on one hand, and between UE and ref on another hand, are noted $\mathbf{h}_{i j}$ and $\mathbf{h}$ ref, respectively, their size being $1 \times M_{f}$. Two steps are required for times $t$ and $t+\Delta t$.

- (i) At time $t$, since the full transfer matrix has been measured, the correlation coefficient $\rho_{i j}$, ref $(t)$ between $\mathbf{h}_{i j}(\mathrm{t})$ and $\mathbf{h}_{\text {ref }}(\mathrm{t})$ can be calculated as:

$$
\rho_{i j, \text { ref }}(t)=\frac{\mathbf{h}_{\text {ref }}(t) \mathbf{h}_{i j}(t)^{H}}{\left\|\mathbf{h}_{\text {ref }}(t)\right\|\left\|\mathbf{h}_{i j}(t)\right\|},
$$

where the upper script $\mathrm{H}$ means conjugate transpose. Applying (1) to the $M_{\text {ref }}$ reference elements, leads to a reduced correlation vector: $\boldsymbol{\rho}(t)=$ $\left[\rho_{i j, 1}, \rho_{i j, 2}, \ldots, \rho_{i j, M \text { ref }}\right]^{T}$.

- (ii) At time $t+\Delta t$, UE sends back an updated estimation of the channel but limited to links between UE and the $M$ ref antennas of the array. This leads to a vector $\mathbf{h}_{\text {ref }}(t+\Delta t)$ of size $\left(1, M_{\text {ref }} M_{f}\right)$.

- (iii) Finally, we suppose that the time interval $\Delta t$ is below the coherence time of the channel. Under this hypothesis, the change in $\boldsymbol{\rho}(t)$ corresponding to the channel second order statistics.

\section{B. Estimation of the channel matrix}

It is proposed to estimate the updated channel vector $\mathbf{h}_{i j}(t+\Delta t)$ between the UE and any array element $(i, j)$ from the knowledge of $\mathbf{h}$ ref $(t+\Delta t)$ and $\boldsymbol{\rho}(t)$, by assimilating this process to an autoregressive model which can be solved using the Yule-Walker equations [17]. In the following, to simplify the notation and since there is no ambiguity, the time reference $t$ or $t+\Delta t$ is omitted. The estimate $\hat{\mathbf{h}}_{i j}$ can be written in the following form [17]:

$$
\hat{\mathbf{h}}_{i j}=\sum_{\text {ref }=1}^{M \text { ref }} \boldsymbol{\alpha}_{\text {ref }} \mathbf{h}_{\text {ref }},
$$

The vector $\boldsymbol{\alpha}_{\text {ref }}$ is defined by:

$$
\boldsymbol{\alpha}_{\text {ref }}=\mathbf{X}^{-1} \boldsymbol{\rho},
$$

where $\mathbf{X}$ is the Toeplitz matrix of $\zeta=$ $\left[1, \rho_{i j, 1}, \rho_{i j, 2}, \ldots, \rho_{i j, M_{\text {ref }}-1}\right]$. For a MU configuration with $K$ UEs, this approach is repeated $K$ times. Qualitatively, one can expect that this approach will provide better results if the channel transfer function does not strongly vary from one reference antenna to its nearest one (large correlation value).

\section{OPTIMIZATION OF THE ALGORITHM AND PERFORMANCES}

An analysis on the performance of the method as a function of the number of reference antennas is presented hereafter using the capability to predict the channel capacity $C$ as a figure of merit. Either individual links to successive UEs, or 
a global link to multi UEs are envisaged. In this last case the sum-rate capacity is calculated using two pre-processing techniques: Maximum Ratio Transmission (MRT) and Zero Forcing (ZF). The channel characteristics used to validate the approach are those deduced from experimental data, as described in Sections II and III. Since measurements have been made in static conditions, the efficiency of the proposed method is based on a comparison between results performed with the full measured matrix to those calculated with the estimated matrix.

\section{A. Single user configuration}

The ergodic capacity for any user $k$, assuming equal power allocation of the $M_{t}$ array antennas, and deduced from the measured channels is given by (4):

$$
C(k)=\log _{2}\left(\operatorname{det}\left(1+\frac{\sigma}{M_{t}} \mathbf{h}_{k} \mathbf{h}_{k}^{H}\right)\right),
$$

where $\sigma$ is the mean signal to noise ratio and $\mathbf{h}_{k}$ is a vector of size $\left(1, M_{t} M_{f}\right)$. The capacity $\hat{C}(k)$ calculated from the estimated channels is obtained by replacing $\mathbf{h}_{k}^{H}$ by $\hat{\mathbf{h}}_{k}^{H}$ in 4). The ratio between $\hat{C}(k)$ and $C(k)$, noted $\beta=\frac{\hat{C}(k)}{C(k)} \times 100$ provides a performance metric for the proposed strategy. Fig. 3 shows $\beta$ as a function of the UE position for $M$ ref values varying from 4 to 32 , and for the $15 \mathrm{Rx}$ positions. In all cases, the reference antennas are uniformly distributed within the array.

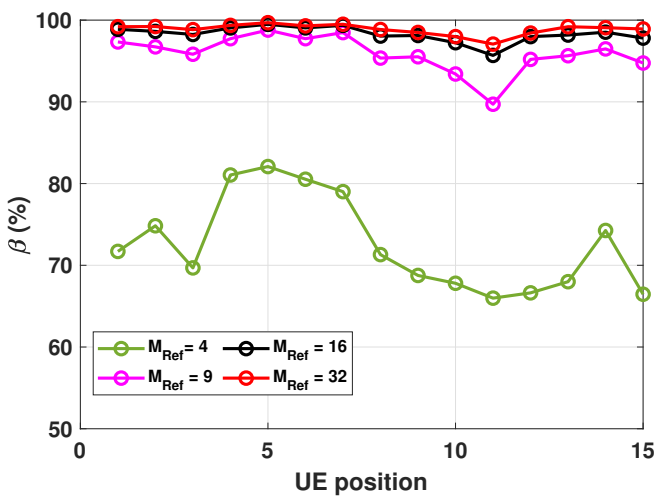

Fig. 3. Impact of the number of reference antennas $M_{\text {ref }}$ on $\beta$ (in $\%$ ) for successive positions of the single user.

First, it is observed in Fig. 3 that the performance of the channel estimator does not strongly depend on the scenarios: LOS (positions 1-2, 4-7), OLOS (3, 12-13) and NLOS (8-11, 14-15). With only 9 reference elements $(\sim 10 \%$ of the total number of Tx antennas), values of $\beta>90 \%$ are reached. The worst case appears for position 11 , having very low spatial coherence amongst antenna elements at the BS. For $M_{\text {ref }}$ $=9$, the separation between 2 successive reference antennas along the $\mathrm{x}$ or $\mathrm{z}$ axis is $3 d$. The correlation median value for this distance evaluated in Section III is $\geq 0.7$. In conclusion, the successive steps to optimize $M$ ref can be the following: (i) From the measured full CSIT matrix at time $t$, calculate the correlation coefficients between all array antennas, (ii) Determine the separation between these antennas, which will be used as reference antennas, such that the median value of $\rho \geq 0.7$, (iii) Equally distribute these reference antennas among the array.

\section{B. Multi-user configuration}

In presence of $K$ simultaneous UEs ( $K=15$ in our application), the sum-rate capacity is given by (5):

$$
C=\log _{2}\left(\operatorname{det}\left(\mathbb{I}_{K}+\frac{\sigma}{M} \mathbf{H W}\right)\right),
$$

$\mathbf{H}$ is the MU-MIMO channel matrix of size $\left(K, M_{t} M_{f}\right)$ and $\mathbf{W}$ the normalized MRT or ZF precoding matrix given in [18] for example. $\beta$ for the sum-rate capacity is given in Table I for a number of reference antennas equal to 4, 9, 16 and 32 .

TABLE I

$\beta(i n \%)$ FOR DIFFERENT NUMBERS OF REFERENCE ANTENNAS, AND FOR ZF AND MRT TECHNIQUES

\begin{tabular}{|c|c|c|c|c|}
\hline$M_{\text {ref }}$ & 4 & 9 & 16 & 32 \\
\hline MRT & 65 & 92 & 93 & 95 \\
\hline ZF & 58 & 80 & 85 & 87 \\
\hline
\end{tabular}

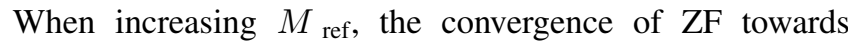
$100 \%$ is slower than for MRT, ZF being more affected by estimation errors. However, it must be outlined that this does not mean that MRT have higher sum-rate capacities compared to those of $\mathrm{ZF}$, at least for the geometrical configuration presented in Fig. 1. Indeed, the capacity deduced from measurements of the full transfer matrix, without estimation error, reaches 29 $\mathrm{bps} / \mathrm{Hz}$ and $89 \mathrm{bps} / \mathrm{Hz}$ for MRT and $\mathrm{ZF}$, respectively. This can be explained by the relatively high number of LOS scenarios (7 among 15). For this case, correlation between these UEs is rather high, with a median value of 0.73 , and the MRT precoding technique does not take inter-user interference into account. From Table I it appears that $M_{\text {ref }}=9$ is an adequate value. Consequently, this validates the approach to select the number of reference antennas previously introduced.

\section{CONCLUSION}

A strategy to reduce the amount of overhead data needed for periodic channel estimation was proposed for FDD schemes. It is based on measurements of massive MIMO transfer functions between UEs and only a few number of array elements, strongly decreasing the size of the channel matrix. The estimation of the full matrix is then obtained owing to the prior knowledge of the correlation matrix at the transmitter. It is demonstrated that the size of the updated channel matrix to be measured can be reduced by a factor of 10 . It must be emphasized that the proposed method is efficient to implement and does not require exhaustive operations for channel estimation. Indeed, the use of a reduced correlation vector of size $\left(1 \times M_{\text {ref }}\right)$ instead of the full matrix simplifies the structure of the Toeplitz matrix and its subsequent inversion. These preliminary results are encouraging and the proposed approach should be further assessed in the context of high-speed user mobility from measured time-varying radio channels. 


\section{REFERENCES}

[1] A. Osseiran, F. Boccardi, V. Braun, K. Kusume, P. Marsch, M. Maternia, O. Queseth, M. Schellmann, H. Schotten, H. Taoka, H. Tullberg, M. A. Uusitalo, B. Timus, and M. Fallgren, "Scenarios for 5G mobile and wireless communications: the vision of the METIS project," IEEE Communications Magazine, vol. 52, no. 5, pp. 26-35, May 2014

[2] A. Gupta and R. K. Jha, "A Survey of 5G Network: Architecture and Emerging Technologies," IEEE Access, vol. 3, pp. 1206-1232, 2015.

[3] T. L. Marzetta, "Noncooperative Cellular Wireless with Unlimited Numbers of Base Station Antennas," IEEE Transactions on Wireless Communications, vol. 9, no. 11, pp. 3590-3600, November 2010.

[4] X. Gao, O. Edfors, F. Rusek, and F. Tufvesson, "Linear Pre-Coding Performance in Measured Very-Large MIMO Channels," in Vehicular Technology Conference (VTC Fall), 2011 IEEE, Sept 2011, pp. 1-5.

[5] Y. Zhang, R. Yu, M. Nekovee, Y. Liu, S. Xie, and S. Gjessing, "Cognitive machine-to-machine communications: visions and potentials for the smart grid," IEEE Network, vol. 26, no. 3, pp. 6-13, May 2012.

[6] CISCO, Cisco Visual Networking Index: Global Mobile Data Traffic Forecast Update, 2016-2021. [Online]. Available: https://www.cisco.com/c/en/us/solutions/collateral/service-provider/ visual-networking-index-vni/mobile-white-paper-c11-520862.pdf

[7] S. K. Rao and R. Prasad, "Impact of 5G Technologies on Industry 4.0," Wirel. Pers. Commun., vol. 100, no. 1, pp. 145-159, May 2018. [Online]. Available: https://doi.org/10.1007/s11277-018-5615-7

[8] A. Adhikary, J. Nam, J. Ahn, and G. Caire, "Joint Spatial Division and Multiplexing-The Large-Scale Array Regime," IEEE Transactions on Information Theory, vol. 59, no. 10, pp. 6441-6463, Oct 2013.

[9] J. Nam, J. Ahn, A. Adhikary, and G. Caire, "Joint spatial division and multiplexing: Realizing massive MIMO gains with limited channel state information," in 2012 46th Annual Conference on Information Sciences and Systems (CISS), March 2012, pp. 1-6.

[10] J. Nam, A. Adhikary, J. Ahn, and G. Caire, "Joint Spatial Division and Multiplexing: Opportunistic Beamforming, User Grouping and Simplified Downlink Scheduling," IEEE Journal of Selected Topics in Signal Processing, vol. 8, no. 5, pp. 876-890, Oct 2014.

[11] W. Shen, L. Dai, B. Shim, S. Mumtaz, and Z. Wang, "Joint csit acquisition based on low-rank matrix completion for fdd massive mimo systems," IEEE Communications Letters, vol. 19, no. 12, pp. 2178-2181, Dec 2015.

[12] X. Rao and V. K. N. Lau, "Distributed Compressive CSIT Estimation and Feedback for FDD Multi-User Massive MIMO Systems," IEEE Transactions on Signal Processing, vol. 62, no. 12, pp. 3261-3271, June 2014.

[13] P. Kuo, H. T. Kung, and P. Ting, "Compressive sensing based channel feedback protocols for spatially-correlated massive antenna arrays," in 2012 IEEE Wireless Communications and Networking Conference (WCNC), April 2012, pp. 492-497.

[14] J. Choi, D. J. Love, and P. Bidigare, "Downlink Training Techniques for FDD Massive MIMO Systems: Open-Loop and Closed-Loop Training With Memory," IEEE Journal of Selected Topics in Signal Processing, vol. 8, no. 5, pp. 802-814, Oct 2014.

[15] J. Joung, E. Kurniawan, and S. Sun, "Channel Correlation Modeling and its Application to Massive MIMO Channel Feedback Reduction," IEEE Transactions on Vehicular Technology, vol. 66, no. 5, pp. 3787-3797, May 2017.

[16] E. Tanghe, D. P. Gaillot, M. Liénard, L. Martens, and W. Joseph, "Experimental Analysis of Dense Multipath Components in an Industrial Environment," IEEE Transactions on Antennas and Propagation, vol. 62, no. 7, pp. 3797-3805, July 2014.

[17] J. A. Cadzow, "Spectral estimation: An overdetermined rational model equation approach," Proceedings of the IEEE, vol. 70, no. 9, pp. 907939, Sep. 1982.

[18] H. Q. Ngo, H. A. Suraweera, M. Matthaiou, and E. G. Larsson, "Multipair full-duplex relaying with massive arrays and linear processing," IEEE Journal on Selected Areas in Communications, vol. 32, no. 9, pp. 1721-1737, Sep. 2014. 